\title{
Association between the ornithine decarboxylase G316A polymorphism and breast cancer survival
}

\author{
LINPING XU $^{1 *}$, JIANPING LONG $^{2 *}$, PENG WANG $^{3}$, KANGDONG LIU $^{1}$, LING MAI $^{1}$ and YONGJUN GUO ${ }^{1}$ \\ ${ }^{1}$ Department of Scientific Research and Foreign Affairs, The Affiliated Cancer Hospital of Zhengzhou University, \\ Zhengzhou, Henan 450000; ${ }^{2}$ Department of Breast Surgery, \\ Maternity and Child-Care Hospital of Gansu Province, Lanzhou, Gansu 730050; \\ ${ }^{3}$ Henan Key Laboratory of Tumor Epidemiology, College of Public Health, \\ Zhengzhou University, Zhengzhou, Henan 450001, P.R. China
}

Received June 5, 2014; Accepted February 19, 2015

DOI: $10.3892 / \mathrm{ol} .2015 .3201$

\begin{abstract}
Ornithine decarboxylase (ODC) is a significant rate-limiting enzyme in polyamine synthesis, required for normal cell growth, and is highly expressed in various malignancies, including colorectal and breast cancer. In the present study, the associations between the ODC G316A single nucleotide polymorphism (SNP) and breast cancer-specific survival were investigated. In addition, the functional effects of this SNP were examined in the MCF-7 human breast cancer cell line. The present study recruited 300 stage I-III breast cancer cases, which were diagnosed at the Affiliated Cancer Hospital of Zhengzhou University (Zhengzhou, China) between 2002 and 2003, with follow-up visits conducted until May 2013. ODC G316A was genotyped (ODC GG vs. ODC AG/AA) in the 300 cases and the association of the genotypes with cancer-specific survival was analyzed. In the MCF-7 cell line, the ODC allele-specific binding of E-box transcription factors was determined using western blot and chromatin immunoprecipitation assays. Survival differences were observed between the two genotypes: Compared with the ODC GG genotype, patients with ODC GA/AA exhibited significantly higher survival rates $(\mathrm{P}<0.05)$. In cultured cells, the ODC SNP, which is flanked by two E-boxes, appeared to predict ODC promoter activity. Furthermore, the E-box activator c-MYC and repressor MAX interactor 1 were found to preferentially bind to ODC minor A-alleles compared with major G-alleles, in cultured MCF-7 cells. In conclusion, the results of the current
\end{abstract}

Correspondence to: Professor Ling Mai or Professor Yongjun Guo, Department of Scientific Research and Foreign Affairs, The Affiliated Cancer Hospital of Zhengzhou University, 127 Dong Ming Road, Zhengzhou, Henan 450003, P.R. China

E-mail: mailing65588018@163.com

E-mail: yongjunguo@hotmail.com

*Contributed equally

Key words: polymorphism, breast cancer, ornithine decarboxylase G316A, MCF-7 cells study suggest that the regulation of ODC may affect survival in breast cancer patients and indicate a model in which the ODC SNP may be protective for breast adenoma recurrence and detrimental for survival following a diagnosis of breast cancer.

\section{Introduction}

Breast cancer is the most common malignancy in females worldwide and its incidence has increased rapidly over recent decades (1). It is well-established that polyamines are low molecular weight, positively-charged compounds that are highly-expressed in numerous malignancies, including breast cancer (2). Previous studies have indicated that polyamines may affect various processes in transcription, RNA stabilization, ion channel gating and carcinogenesis, while inhibition of polyamine synthesis appears to be important in inhibiting proliferation, decreasing apoptosis and suppressing angiogenesis (3-5). Ornithine decarboxylase (ODC) is a rate-limiting enzyme that contributes to polyamine synthesis (6). Increased polyamine levels and ODC activity have been identified in various types of human cancer, particularly in breast cancer. Therefore, inhibiting ODC activity was able to suppress cancer development in animal models (7-10). ODC expression is regulated by the MYC and MAD transcription factors, which include the MYC-associated factor X (MAX) network transcriptional repressor, MAX interactor 1 (MXI1), MAD1 and MAD4 (11). When MYC forms a heterodimer with MAX, it can bind to DNA at the E-box sequence (CACGTG) and activate ODC gene expression. By contrast, MAD combines with MAX, which can suppress the transcription of the ODC gene (Fig. 1) (12,13).

ODC G316A is a functional single nucleotide polymorphism (SNP) that is located between two promoter region/transcription factor binding sites. Furthermore, the transcription of the ODC gene can be altered by this SNP (14). Previous studies have demonstrated that the ODC G316A polymorphism exhibits prognostic efficacy in colorectal adenoma recurrence and is associated with the survival of patients with colorectal cancer $(15,16)$. However, the effects of this SNP on the clinical outcomes in breast cancer remain unclear. Therefore, the aim of the present population-based study was to 
investigate the association between the ODC G316A SNP and breast cancer-specific mortality by genotyping 300 samples from breast cancer patients that were enrolled at the Affiliated Cancer Hospital of Zhengzhou University (Zhengzhou, China) between 2002 and 2003. Subsequently, the functional significance of the ODC G316A SNP in the MCF-7 breast cancer cell line was investigated.

\section{Materials and methods}

\section{Epidemiological studies}

Study population. The present study investigated 300 patients with stage I-III breast cancer that were enrolled at the Affiliated Cancer Hospital of Zhengzhou University between 2002 and 2003, with follow-up visits conducted until May 2013. For all breast cancer patients recruited to the present study, written informed consent was obtained from the patients or the patient's families, allowing the collection of paraffin-embedded tissue samples and the release of the patients' medical data. Furthermore, the present study was approved by the Ethics Committee of the Affiliated Cancer Hospital of Zhengzhou University. Clinical and demographic data, including vital status and follow-up details, were obtained by associating follow-up and regional cancer registry databases. Information regarding lifestyle factors and a detailed family history were collected from all the study participants during face-to-face interviews. Tumor grade was determined according to the 2013 breast cancer guidelines of the National Comprehensive Cancer Network (17). The morphological features (tubule formation, nuclear pleomorphism and mitotic count) of the tumors were assessed and each assigned a score of 1-3. The scores for each category were combined to determine the histological grades of the tumors as follows: Grade 1 (low), score, 3-5; grade 2 (intermediate), score, 6-7; and grade 3 (high), score, 8-9. The 'tumor node metastasis' staging was derived from existing American Joint Committee on Cancer (AJCC) codes, where available, and conversion of the extent of disease codes was performed as previously reported $(18,19)$.

DNA extraction. Subsequently, DNA was extracted from 300 paraffin-embedded breast cancer tissue samples obtained during surgery between May and June 2013, which were previously stored at the Affiliated Cancer Hospital of Zhengzhou University, using a QIAamp DNA FFPE Tissue kit (Qiagen, Hilden, Germany) (20), according to the manufacturer's instructions.

Primer design. The ODC sequence was obtained from GenBank (accession no. NC_009142.1) and polymerase chain reaction (PCR) primers were designed using the Premier Primer software (version 5.0; Premier Biosoft, Palo Alto, CA, USA). The forward (F) 1 and reverse (R) 1 primers were used to amplify a 547-bp region, while the F2 and R2 primers were used amplify a 178-bp region within the first 547-bp amplification. The two PCR primer pairs are indicated in Table I.

Nested PCR-restriction fragment length polymorphism (RFLP). ODC G316A genotypes were generated using nested PCR-RFLP technology. DNA amplification was performed in a $25-\mu 1$ reaction volume consisting of $12.5 \mu 12 \mathrm{X}$ PCR buffer for KOD FX (Toyobo Co., Ltd., Osaka, Japan),
Table I. Oligonucleotide primers used to amplify the ornithine decarboxylase gene.

\begin{tabular}{llc}
\hline Primer & \multicolumn{1}{c}{ Sequence } & $\begin{array}{c}\text { Size of } \\
\text { DNA } \\
\text { product, bp }\end{array}$ \\
\hline F1 & 5'-GGTGCTATAAGTAGGGAGCGGC-3' & 547 \\
R1 & 5'-CGAAGGGTTGGGAAAGAGGC-3' & 547 \\
F2 & 5'-CCTGGGGGCTCTGAGGT-3' & 178 \\
R2 & 5'-AGGAAGCGGCGCCTCAA-3' & 178 \\
\hline
\end{tabular}

F, forward; R, reverse.

$5 \mu 12 \mathrm{mM}$ deoxynucleotide triphosphate, $2 \mu \mathrm{l}$ of each primer, $0.1 \mu 1 \mathrm{KOD}, 2.4 \mu \mathrm{l}$ water and $1 \mu \mathrm{l} \mathrm{DNA}$. The standard conditions for PCR were as follows: $95^{\circ} \mathrm{C}$ for $2 \mathrm{~min}$; followed by 40 cycles each at $95^{\circ} \mathrm{C}$ for $30 \mathrm{sec}$ and $62^{\circ} \mathrm{C}$ for $1 \mathrm{~min}$; and a final extension at $72^{\circ} \mathrm{C}$ for $5 \mathrm{~min}$. All the reactions were performed in a Perkin Elmer 2400 thermocycler (Perkin Elmer, Foster City, CA, USA). The nested PCR products were analyzed by detecting the polymorphic PstI site using capillary electrophoresis (QIAxcel Advanced System; Qiagen).

\section{Experimental studies}

Cell culture. MDA-435 cells and the MCF-7 human breast cancer cell line, which exhibited an ODC AG genotype, were maintained in Dulbecco's modified Eagle's medium (Invitrogen Life Technologies, Carlsbad, CA, USA) and all media used were supplemented with $10 \%$ fetal bovine serum. Furthermore, cells were maintained at $37^{\circ} \mathrm{C}$ in a humidified atmosphere of $5 \% \mathrm{CO}_{2}$.

Western blot analysis. Cells were harvested and lysed, and proteins were separated on a $12.5 \%$ SDS-PAGE gel (15). Next, the proteins were transferred by electrophoresis onto a Hybond-C membrane (GE Healthcare Life Sciences, Little Chalfont, UK), which was blocked with Blotto A (5\% blocking grade dry milk in Tris-buffered saline/Tween-20; Santa Cruz Biotechnology, Inc., Dallas, TX, USA), and probed using monoclonal mouse anti-human c-MYC (1:300; cat. no. sc-41; Santa Cruz Biotechnology, Inc.) and monoclonal mouse anti-human anti-MXI1 (1:300; cat. no. sc-130627; Santa Cruz Biotechnology, Inc.) primary antibodies in Blotto A. The primary antibodies were incubated at $4^{\circ} \mathrm{C}$ overnight, followed by incubation with a monoclonal goat anti-mouse $\mathrm{Ig}$ horseradish peroxidase-tagged secondary antibody (1:1,000; cat. no. sc-2005; Santa Cruz Biotechnology, Inc.) for $1 \mathrm{~h}$ at room temperature. Chemiluminescent detection was conducted using an electrochemiluminescence western blotting detection reagent (GE Healthcare Life Sciences) and exposing on a Biomax XAR film (Kodak, Rochester, NY, USA).

Chromatin immunoprecipitation (ChIP). ChIP assays were performed using a ChIP Assay Kit, according to the manufacturer's instructions (Cell Signaling Technology, Inc., Danvers, MA, USA). Briefly, the cells were treated with $1 \%$ formaldehyde to cross-link the DNA and proteins prior to disruption of the DNA-protein complexes by sonication [25\% ultrasound; 14 cycles (4.5 sec each)] to produce fragment 
Table II. Descriptive analysis for breast cancer cases overall and based on ODC genotype.

\begin{tabular}{|c|c|c|c|c|}
\hline \multirow[b]{2}{*}{ Category } & \multirow{2}{*}{$\begin{array}{l}\text { Total breast cancer } \\
\text { cases }(\mathrm{n}=300), \mathrm{n}(\%)\end{array}$} & \multicolumn{2}{|c|}{ ODC G316A genotype } & \multirow[b]{2}{*}{ P-value } \\
\hline & & $\mathrm{GG}(\mathrm{n}=156), \mathrm{n}(\%)$ & GA/AA (n=144), n (\%) & \\
\hline Age, years ${ }^{a}$ & & & & 0.280 \\
\hline$\leq 45$ & $125(41.67)$ & $68(43.59)$ & $57(39.58)$ & \\
\hline$>45$ & $175(58.33)$ & $88(56.41)$ & $87(60.42)$ & \\
\hline Stage at diagnosis & & & & 0.482 \\
\hline I & $90(30.00)$ & 48 (30.77) & $42(29.17)$ & \\
\hline II & $108(36.00)$ & $56(35.90)$ & $52(36.11)$ & \\
\hline III & $102(34.00)$ & $52(33.33)$ & $50(34.72)$ & \\
\hline Family history of cancer & & & & 0.948 \\
\hline Yes & $106(35.33)$ & $54(34.62)$ & $52(36.11)$ & \\
\hline No & $194(64.67)$ & $102(65.38)$ & $92(63.89)$ & \\
\hline Tumor grade & & & & 0.787 \\
\hline 1 & $64(21.33)$ & $35(22.44)$ & $29(20.14)$ & \\
\hline 2 & $191(63.67)$ & $97(62.18)$ & $94(65.28)$ & \\
\hline 3 & $45(15.00)$ & $24(15.38)$ & $21(14.58)$ & \\
\hline ER and PR status & & & & 0.848 \\
\hline Positive & $187(62.33)$ & $99(63.46)$ & $88(61.11)$ & \\
\hline Negative & $113(37.67)$ & $57(36.54)$ & $56(38.89)$ & \\
\hline Her-2 status & & & & 0.675 \\
\hline Positive & $128(42.67)$ & $65(41.67)$ & $63(43.75)$ & \\
\hline Negative & $172(57.33)$ & $91(58.33)$ & $81(56.25)$ & \\
\hline Ki-67 status & & & & 0.715 \\
\hline Positive & $167(55.67)$ & 85 (54.49) & $82(56.94)$ & \\
\hline Negative & $133(44.33)$ & $71(45.51)$ & $62(43.05)$ & \\
\hline Surgical treatment & & & & 0.669 \\
\hline Yes & $300(100.00)$ & $156(100.00)$ & $144(100.00)$ & \\
\hline No & $0(0.00)$ & $0(0.00)$ & $0(0.00)$ & \\
\hline Radiation therapy & & & & 0.963 \\
\hline Yes & $165(55.00)$ & $86(55.12)$ & $79(54.86)$ & \\
\hline No & $135(45.00)$ & $70(44.87)$ & $65(45.14)$ & \\
\hline Chemotherapy & & & & 0.981 \\
\hline Yes & $246(82.00)$ & $128(82.05)$ & $18(81.94)$ & \\
\hline No & $54(18.00)$ & $28(17.95)$ & $26(18.06)$ & \\
\hline
\end{tabular}

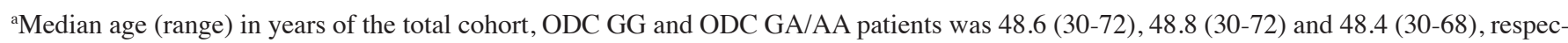
tively. ODC, ornithine decarboxylase; ER, estrogen receptor; PR, progesterone receptor; Her-2, human epidermal growth factor receptor 2.

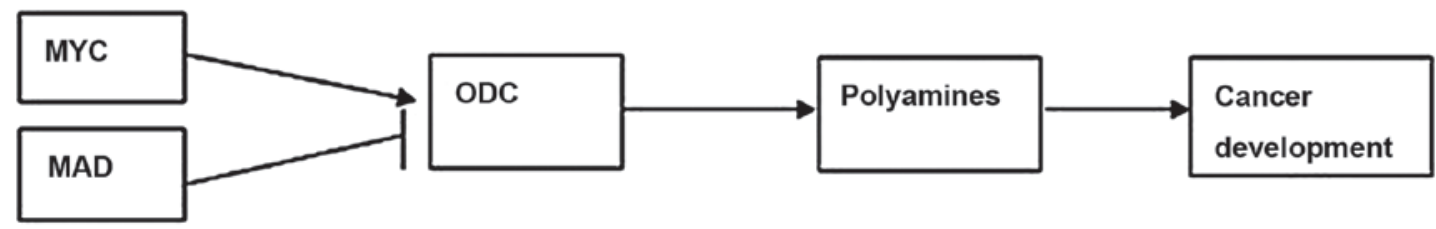

Figure 1. Schematic diagram depicting the differential effects of polyamine regulation by MAD and MYC on ODC. ODC, ornithine decarboxylase.

lengths of 200-1,000 bp. The lysates were then diluted 10 times with immunoprecipitation dilution buffer containing $1 \mathrm{mmol} / 1$ phenylmethanesulfonyl fluoride. Antibodies for c-MYC and MXI1 (Santa Cruz Biotechnology, Inc.) were used to treat the samples, in order to induce chromatin precipitation. However, one sample was left untreated as a minus-antibody control. Immunoprecipitation was performed overnight at $4^{\circ} \mathrm{C}$ with centrifugation at $234.8 \times \mathrm{g}$ for $15 \mathrm{~min}$. 
Table III. Breast cancer-specific survival analysis of patients with breast cancer based on the ODC G316A genotype.

Breast cancer-specific mortality

ODC G316A genotype

Events, $\mathrm{n} \quad$ At risk, $\mathrm{n}$

$\operatorname{HR}(95 \% \mathrm{CI})^{\mathrm{a}}$

\section{GG}

GA/AA
32

40
156

144
1 (reference)

$1.57(1.16-3.34)$

${ }^{\mathrm{a}} \mathrm{P}=0.037$. ODC, ornithine decarboxylase; HR, hazard ratio; CI, confidence interval.

Subsequently, immune complexes were obtained by adding $60 \mu 1$ salmon sperm DNA/protein A agarose slurry (Merck Millipore, Darmstadt, Germany) and incubating for $1 \mathrm{~h}$ at $4^{\circ} \mathrm{C}$ with rotation, followed by gentle centrifugation (335.4 $\mathrm{x}$ g for $1 \mathrm{~min})$. Protein A agarose pellets were washed with low-salt, high-salt, $\mathrm{LiCl}$ and Tris-EDTA buffer. The complexes were then eluted twice by adding $250 \mu \mathrm{l}$ elution buffer $(0.1 \mathrm{~mol} / 1$ $\mathrm{NaHCO}_{3}$ and $1 \% \mathrm{SDS}$ ), and DNA-protein cross-links were reversed using $0.2 \mathrm{~mol} / 1 \mathrm{NaCl}$ and heating at $65^{\circ} \mathrm{C}$ for $4 \mathrm{~h}$ for all the samples, including the input DNA and minus-antibody DNA controls. Finally, DNA was re-suspended in $30 \mu \mathrm{l}$ double-distilled $\mathrm{H}_{2} \mathrm{O}$.

Statistical analysis. All the statistical data were analyzed using SPSS statistical software (version 17.0; SPSS Inc., Chicago, IL, USA). A $\chi^{2}$ test was used to describe the breast cancer cases overall and in regard to their ODC genotype. Survival curves were plotted using the Kaplan-Meier method and analyzed using the log-rank test. Furthermore, Cox's proportional hazards model was used to identify prognostic factors for survival. For all data, $\mathrm{P}<0.05$ was considered to indicate a statistically significant difference.

\section{Results}

\section{Epidemiological study}

Clinicopathological characteristics of breast cancer based on ODC G316A SNP genotypes. In order to enhance sensitivity, nested PCR was used to amplify the products. The first set of primers were designed to amplify a 547-bp region within the ODC gene, while the second primer pair was designed to amplify a 178-bp region within the 547-bp region, and these were used in the nested PCR assay. Capillary electrophoresis technology was subsequently employed to determine the sequence of the PCR products. For the 178-bp region of the ODC gene, Pst I enzyme digestion and PCR-RFLP were used to genotype the ODC G316A SNP. The results of capillary electrophoresis were termed C1-C300. For the ODC AG genotype, the 178, 50 and 120-bp regions were obtained. However, only a single region (178-bp) was identified for ODC GG, whereas the 50 and 20-bp regions were detected for ODC AA. A total of 300 stage I-III breast cancer cases admitted at the Affiliated Cancer Hospital of Zhengzhou University were used in the present case-only analysis. Nested PCR-RFLP was used to genotype the patients, and the median follow-up duration was 10 years and 3 months. The cohort included 156 (52\%) ODC GG and $144(48 \%)$ ODC GA/AA cases. Clinicopathological data for

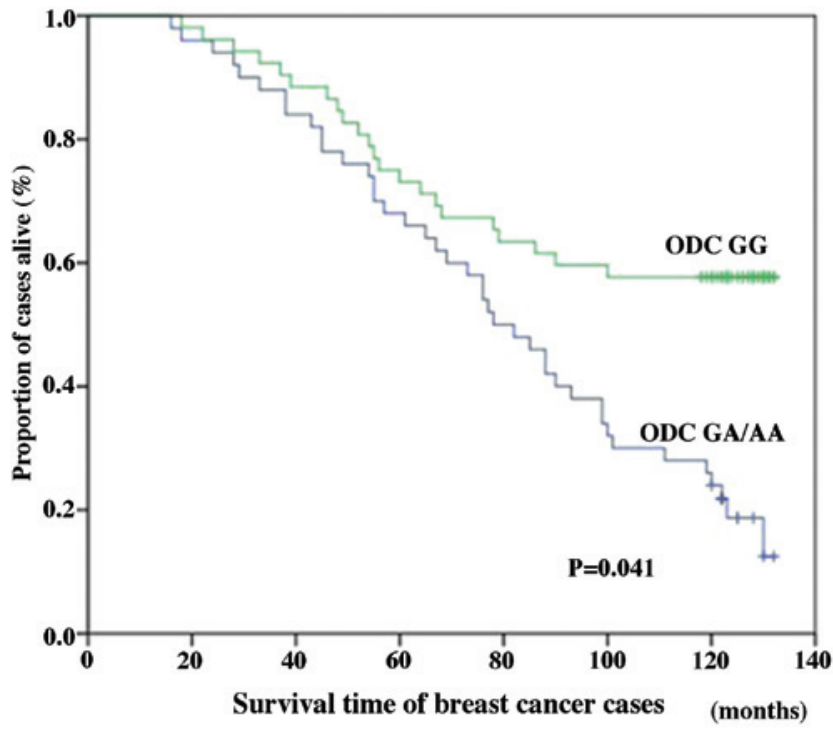

Figure 2. Kaplan-Meier demonstrating the breast cancer-specific survival rate estimates for cases with stage III breast cancer, stratified by ODC G316A genotype. The study included 300 cases of breast cancer, which were enrolled between 2002 and 2003, with follow-up visits conducted until May 2013. The number of breast cancer-specific mortalities in patients carrying the ODC GG and GA/AA phenotypes were 32 and 40, respectively. ODC, ornithine decarboxylase.

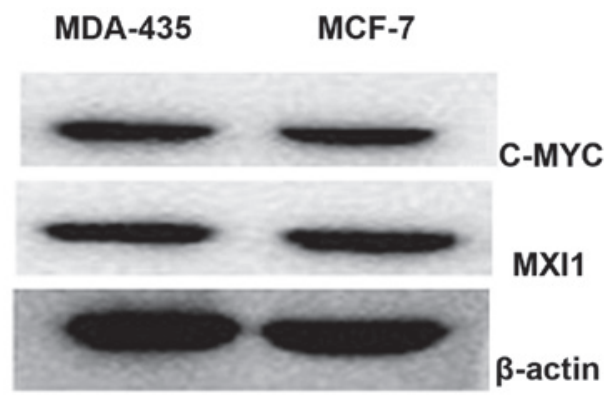

Figure 3. E-box protein expression levels in breast cancer-derived cells. The expression of protein binding at the +316 bp ODC single nucleotide polymorphism was assessed by western blot analysis. Extracts of MDA-435 and MCF-7 cells were evaluated for c-MYC and MXI1 expression, and $\beta$-actin was used as the loading control. MXI1, MAX interactor 1.

the breast cancer cases based on ODC genotype are indicated in Table II.

Cancer-specific survival time based on ODC G316A genotypes. Of the 300 stage I-III breast cancer cases, 168 patients $(56 \%)$ had succumbed to the disease prior to the analysis. In total, 72 mortalities (42.86\%) occurred in 


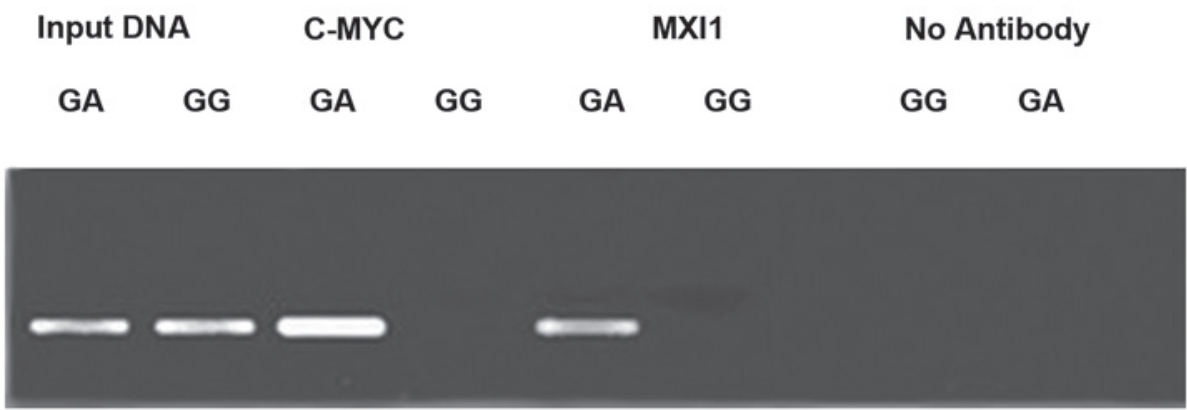

Figure 4. Documentation of allele-specific transcription factor binding by chromatin immunoprecipitation analysis, which was conducted as described in Materials and Methods. MCF-7 cells were a source of ornithine decarboxylase (ODC). A-alleles, as these cells are heterozygous GA at this site. MDA-435 cells were used as a source of ODC G-alleles. MXI1, MAX interactor 1.

patients carrying the ODC GG genotype, compared with 96 mortalities (57.14\%) in patients with the GA/AA genotypes. A statistically significant improvement in breast cancer-specific survival was observed among breast cancer cases homozygous for the ODC G-allele (10-year survival, 53.85\%) compared with cases exhibiting at least one A-allele (ODC GA/AA; 10 -year survival, 33.33\%; $\mathrm{P}<0.001)$. Furthermore, breast cancer-specific survival analysis by stage revealed no statistically significant differences in the survival of patients with AJCC stages I ( $\mathrm{P}=0.537)$ or II $(\mathrm{P}=0.482$; data not shown). However, among cases of stage III breast cancer, the ODC GG genotype was significantly associated with improved 10 -year breast cancer-specific survival $(38.46 \%)$ compared with the ODC GA/AA genotype cases (20.00\%; $\mathrm{P}=0.041 ;$ Fig. 2$)$. Among all the cases (stages I-III), differences in the genotype-specific breast cancer survival were statistically significant, with the ODC G316A SNP acting as an independent predictor of breast cancer-specific survival. Compared with the ODC GG genotype cases [hazard ratio (HR), 1; 95\% confidence interval (CI), 1, reference], the breast cancer-specific risk of mortality was significantly greater for the ODC GA/AA genotype (HR, 1.57; 95\% CI, 1.16-4.23; P=0.037; Table III).

\section{Experimental studies}

Western blot analysis. The expression of specific E-box binding proteins, including the transcriptional activator $\mathrm{c}-\mathrm{MYC}$ and the transcriptional repressor MXI1, was established by western blotting of MCF-7 cell proteins (Fig. 3). c-MYC and MXI1 were expressed in the MDA-435 and MCF-7 cells (Fig. 3).

ChIP. To investigate the affinity of the c-MYC and MXI1 proteins for the ODC G316A allele, ChIP analysis of the $+316 \mathrm{bp}$ region of the ODC promoter was conducted using antibodies against the aforementioned E-box binding proteins (Fig. 4). The results revealed that protein bands for the c-MYC and MXI1 proteins were present in the MCF-7 cell lines, but not in the MDA-435 cell lines. These results indicate that c-MYC and MXI1 proteins may selectively bind to ODC G316A allele A, rather than the $\mathrm{G}$.

\section{Discussion}

Accumulating evidence has indicated that polyamine catabolism is involved in the response to therapeutic agents, apoptosis and the stress response, and is important in the development of psoriasis, parasitic infection and cancer (21-23). In recent years, a number of anticancer compounds relevant to polyamine biosynthetic single enzyme inhibitors have been developed, including the $\alpha$-difluoromethylornithine and methylglyoxal bis-guanylhydrazone. However, the application of these compounds in a clinical setting is limited due to their poorly-tolerated adverse reactions $(24,25)$. Ornithine decarboxylase (ODC) is the most significant and rate-limiting enzyme in the pathway of polyamine synthesis, which is critical in cell proliferation and highly expressed in a variety of cancer types, including breast cancer $(6,26-28)$. Previous studies have indicated that the overexpression of ODC is common in carcinogenesis and cancer progression (6,26-28). Therefore, certain studies have proposed that it may be an important biological marker in the evaluation of biological behavior and the prognosis of various types of cancer $(13,26)$. Recently, an increasing number of studies have focused on ODC as a potential target for cancer therapy $(24,25)$. In particular, ODC has been proposed to be a promising candidate target for natural products in cancer chemoprevention (29). Thus, future investigation of ODC inhibitors present in nature may facilitate the identification of novel cancer chemopreventive agents. In 2000, Guo et al (14) identified a single nucleotide polymorphism (SNP) in the gene regulatory region of ODC, known as ODC G316A. This SNP is located between two significant gene regulatory regions, namely the MYC/MAX/MAD protein binding region, CACGTG E-box, which is known to regulate ODC transcription. However, only a limited number of studies have focused on the association between the ODC G316A genotype and breast cancer survival, as well as the underlying mechanism. In 2003, Martinez et al (30) reported that the ODC G316A polymorphism was able to independently reduce the risk of adenoma recurrence by inhibiting synthesis and activating catabolism of the tumor cells. Furthermore, in colorectal cancer, the ODC polymorphism appeared to act as a genetic marker for predicting colon cancer recurrence. Furthermore, the ODC G316A genotype was found to be a prognostic factor in colorectal adenoma recurrence and survival $(15,16)$.

However, thus far, no evidence exists to indicate that this SNP exhibits the same function in breast cancer as in colorectal cancer. In 2009, Brown et al (31) reported that the ODC G316A polymorphism (SNP no. rs2302615) may be less important in individuals with an inherited predisposition 
for breast cancer than in individuals who develop sporadic breast cancer (32). However, our study involved the ODC G316A polymorphism (SNP no. rs1045900). Therefore, the present study used nested-PCR-RFLP to genotype the ODC G316A SNP in breast cancer. Of the 300 cases of breast cancer investigated, 156 were ODC GG cases and 144 were ODC GA/AA cases. Additional analysis demonstrated that patients with the GG phenotype exhibited significantly higher 10-year survival rates compared with those with the GA/AA phenotype. Furthermore, according to the results of the present study, patients with an A allele (ODC GA/AA patients) exhibited significantly lower 10 -year survival rates compared with patients presenting the ODC GG phenotype, indicating that the ODC G316A SNP may be a useful marker for predicting the survival of patients with breast cancer. The results of the present study were in accordance with the report by Hubner et al (15); however, the present study was conducted in a Chinese population and used the simpler and more economical technology of nested-PCR-RFLP to genotype breast cancer. Overall, the current results indicated that the ODC G316A genotype is associated with breast cancer survival.

The ODC A allele has been reported to be associated with poor survival in colorectal cancer (16). Martinez et al (30) reported that MAD1 (as well as MXI1) selectively suppressed the activity of the ODC promoter containing the A allele, as opposed to the $\mathrm{G}$ allele, in the HT29 human colon cancer-derived cell line. Furthermore, Hubner et al (15) achieved the same results using ChIP in different human colon cancer-derived cell lines (HCT116 and HT29). However, due to insufficient evidence, it was unclear whether the same outcome would occur in human breast cancer-derived cell lines. A previous study of the current group identified that c-MYC and MXI1 protein expression levels are associated with the prognosis of breast cancer patients (32). Furthermore, the present study employed western blotting and ChIP to examine the association between the two E-box proteins and the ODC SNP. In cultured cells, the E-box activator c-MYC and repressor MXI1 were found to preferentially bind to ODC minor A alleles, rather than major $\mathrm{G}$ alleles. Consistent with the results of the present study, Zell et al (16) reported that c-MYC, MAD1 and MAD4 preferred binding to ODC minor A-alleles, rather than major G-alleles, in vitro.

In conclusion, the present study genotyped ODC G316A in patients with breast cancer and investigated the clinical outcome of the ODC G316A SNP on breast cancer-specific mortality. The current results indicated that MXI1 preferentially binds to the ODC A-allele, thus, contributing to breast cancer progression. Furthermore, the findings of the present study indicated that the G316A SNP may be used to assess the risk of progression in patients with breast cancer.

\section{Acknowledgements}

The present study was supported by the Henan Medical Science Foundation (grant no. 201203152) and the National Nature Science Foundation of China (grant no. 81372269). The authors would like to thank The Affiliated Cancer Hospital of Zhengzhou University (Zhengzhou, China) and its staff, and Dr. Xibin Sun (Department of Cancer Prevention,
Henan Cancer Hospital, Zhengzhou, China) for the statistical analysis.

\section{References}

1. DeSantis C, Siegel R, Bandi P and Jemal A: Breast cancer statistics, 2011. CA Cancer J Clin 61: 409-418, 2011.

2. Gerner EW and Meyskens FL Jr: Polyamines and cancer: old molecules, new understanding. Nat Rev Cancer 4: 781-792, 2004

3. Wallace HM: The physiological role of the polyamines. Eur J Clin Invest 30: 1-3, 2000.

4. Babbar N, Ignatenko NA, Casero RA Jr and Gerner EW: Cyclooxygenase-independent induction of apoptosis by sulindac sulfone is mediated by polyamines in colon cancer. $\mathrm{J}$ Biol Chem 278: 47762-47775, 2003.

5. Takigawa M, Enomoto M, Nishida Y, Pan HO, Kinoshita A and Suzuki F: Tumor angiogenesis and polyamines: alpha-difluoromethylornithine, an irreversible inhibitor of ornithine decarboxylase, inhibits B16 melanoma-induced angiogenesis in ovo and the proliferation of vascular endothelial cells in vitro. Cancer Res 50: 4131-4138, 1990.

6. Gödderz D, Schäfer E, Palanimurugan R and Dohmen RJ: The N-terminal unstructured domain of yeast ODC functions as a transplantable and replaceable ubiquitin-independent degron. J Mol Biol 407: 354-367, 2011.

7. Chen Y, Megosh LC, Gilmour SK, Sawicki JA and O'Brien TG: $\mathrm{K} 6 / \mathrm{ODC}$ transgenic mice as a sensitive model for carcinogen identification. Toxicol Lett 116: 27-35, 2000.

8. Young L, Salomon R, Au W, Allan C, Russell P and Dong Q: Ornithine decarboxylase (ODC) expression pattern in human prostate tissues and ODC transgenic mice. J Histochem Cytochem 54: 223-229, 2006.

9. Lan L, Trempus C and Gilmour SK: Inhibition of ornithine decarboxylase (ODC) decreases tumor vascularization and reverses spontaneous tumors in ODC/Ras transgenic mice. Cancer Res 60: 5696-5703, 2000.

10. Guo Y, Cleveland JL and O'Brien TG: Haploinsufficiency for odc modifies mouse skin tumor susceptibility. Cancer Res 65: 1146-1149, 2005.

11. Grinberg AV, Hu CD and Kerppola TK: Visualization of Myc/Max/Mad family dimers and the competition for dimerization in living cells. Mol Cell Biol 24: 4294-4308, 2004.

12. Walhout AJ, Gubbels JM, Bernards R, van der Vliet PC and Timmers HT: c-Myc/Max heterodimers bind cooperatively to the E-box sequences located in the first intron of the rat ornithine decarboxylase (ODC) gene. Nucleic Acids Res 25: 1493-1501, 1997.

13. Funakoshi-Tago M, Sumi K, Kasahara T and Tago K: Critical roles of Myc-ODC axis in the cellular transformation induced by myeloproliferative neoplasm-associated JAK2 V617F mutant. PLoS One 8: e52844, 2013.

14. Guo Y, Harris RB, Rosson D, Boorman D and O'Brien TG: Functional analysis of human ornithine decarboxylase alleles. Cancer Res 60: 6314-6317, 2000.

15. Hubner RA, Muir KR, Liu JF, Logan RF, Grainge MJ and Houlston RS; Members of the UKCAP Consortium: Ornithine decarboxylase G316A genotype is prognostic for colorectal adenoma recurrence and predicts efficacy of aspirin chemoprevention. Clin Cancer Res 14: 2303-2309, 2008.

16. Zell JA, Ziogas A, Ignatenko N, et al: Associations of a polymorphism in the ornithine decarboxylase gene with colorectal cancer survival. Clin Cancer Res 15: 6208-6216, 2009.

17. Theriault RL, Carlson RW and Allred C, et al; National Comprehensive Cancer Network: Breast cancer, version 3.2013: featured updates to the NCCN guidelines. J Natl Compr Canc Netw 11: 753-760, 2013.

18. Harris L, Fritsche H, Mennel R, et al; American Society of Clinical Oncology: American Society of Clinical Oncology 2007 update of recommendations for the use of tumor markers in breast cancer. J Clin Oncol 25: 5287-5312, 2007.

19. Singletary SE, Allred C, Ashley P, et al: Revision of the American Joint Committee on Cancer staging system for breast cancer. J Clin Oncol 20: 3628-3636, 2002.

20. Leen S, Steven VL, Marie DA, et al: Study assessing the quality of quantification of estrogen receptor protein expression by immunohistochemistry and gene expression in breast cancer. Patholog Res Int 2014: 372653, 2014.

21. Casero RA and Pegg AE: Polyamine catabolism and disease. Biochem J 421: 323-338, 2009. 
22. Thomas $\mathrm{T}$ and Thomas TJ: Polyamine metabolism and cancer. J Cell Mol Med 7: 113-126, 2003.

23. Wallace HM and Fraser AV: Inhibitors of polyamine metabolism: review article. Amino Acids 26: 353-365, 2004.

24. Wallace HM and Fraser AV: Inhibitors of polyamine metabolism: review article. Amino Acids 26: 353-365, 2004.

25. Levin VA, Uhm JH, Jaeckle KA, et al: Phase III randomized study of postradiotherapy chemotherapy with alpha-difluoromethylornithine-procarbazine, $\mathrm{N}$-(2-chloroethyl)-N'-cyclohe xyl-N-nitrosurea, vincristine (DFMO-PCV) versus PCV for glioblastoma multiforme. Clin Cancer Res 6: 3878-3884, 2000.

26. Deng X and Pei D: Ornithine decarboxylase and glutamate decarboxylase 65 as prognostic markers of gallbladder malignancy: a clinicopathological study in benign and malignant lesions of the gallbladder. Mol Med Rep 7: 413-418, 2013.

27. Wilson SM, Hawel L III, Pastorian KE and Byus CV: A stable, inducible, dose-responsive ODC overexpression system in human cell lines. Biochim Biophys Acta 1732: 103-110, 2005.
28. Love RR, Astrow SH, Cheeks AM and Havighurst TC: Ornithine decarboxylase (ODC) as a prognostic factor in operable breast cancer. Breast Cancer Res Treat 79: 329-334, 2003.

29. Luqman S: Ornithine decarboxylase: a promising and exploratory candidate target for natural products in cancer chemoprevention. Asian Pac J Cancer Prev 13: 2425-2427, 2012.

30. Martinez ME, O'Brien TG, Fultz KE, et al: Pronounced reduction in adenoma recurrence associated with aspirin use and a polymorphism in the ornithine decarboxylase gene. Proc Natl Acad Sci USA 100: 7859-7864, 2003.

31. Brown I, Halliday S, Greig H, et al: Genetic polymorphism in ornithine decarboxylase and risk of breast cancer. Fam Cancer 8: 307-311, 2009.

32. Xu LP, Sun Y, Li W, Mai L, Guo YJ and Fan QX: MYC and MXI1 protein expression: potential prognostic significance in women with breast cancer in China. Oncol Res Treat 37: 118-123, 2014. 